\title{
Participación Comunitaria: Necesidad, Excusa o Estrategia? 0 de qué Hablamos Cuando Hablamos de Participación Comunitaria
}

\author{
Community Participation: Need, Excuse, or Strategy? What are We \\ Talking About When We Refer to Community Participation?
}

\section{Mario Bronfman ${ }^{1}$ \\ Marcela Gleizer ${ }^{1}$}

BRONFMAN, M. \& GLEIZER, M. Community Participation: Need, Excuse, or Strategy? What are We Talking About When We Refer to Community Participation? Cad. Saúde Públ., Rio de Janeiro, 10 (1): 111-122, Jan/Mar, 1994.

This paper evaluates the strategy of including community participation in health care and health promotion programs, taking into account three dimensions: the pertinence of the practice, the characteristics of its implementation, and the results generated by it.

After doing a critical review of the bibliography on the issue, we came to the conclusion that many heterogeneous programs are grouped under the term "community participation". This requires a specification of what king of community participation is being talked about. As a consequence, the study proposes a classification of the different practices grouped under this heading. The following criteria were used in the classification: 1) theoretical and ideological conceptions underlying the practice of community participation; 2) aspects to be implemented and subjects to be influenced by intervention; and 3) forms that community participation takes in practice. The study also analyzes the articulation between these three aspects.

Finally, the role of social research and qualitative methodology in the implementation of community participation for health care programs is considered. The study also considers challenges that overall proposals must face in order for strategies to develop successfully, beyond their differences in conception and implementation.

Key words: Consumer Participation; Health Promotion

\section{INTRODUCCIÓN}

La inclusión de la participación comunitaria en los programas de salud no es una práctica novedosa. Implica la incorporación de la población o de sectores de ella, en grados y formas variables, a alguna actividad específica relacionada con la salud. Sin embargo, al igual que otros conceptos, el de "participación comunitaria" no denota siempre al mismo objeto. Revisando la literatura sobre el tema, encontramos que bajo el mismo nombre se incluyen:

${ }^{1}$ Secretaría de Salud (Estados Unidos Mexicanos). FCO. de P. Miranda \# 177 Piso 4. Unidad Plateros, 01 480, Mexico, $D F$.
1) distintas concepciones sobre el sentido de la incorporación de la comunidad en el diseño y desarrollo de programas de salud, y

2) distintas formas y niveles en los que la participación comunitaria se lleva a cabo.

Ambos aspectos, como luego trataremos de demostrar, están íntimamente relacionados.

\section{EL SENTIDO DE LA PARTICIPACIÓN COMUNITARIA}

Las posiciones acerca e la inclusión de la participación comunitaria en los programas de salud son diversas e, inclusive, contradictorias. 
Simplificando, podemos distinguir dos posiciones extremas - la que apoya la inclusión de la participación en los programas y la que la rechaza - y dos criterios o dimensiones principales para justificar esas posiciones: el que enfatiza las consecuencias políticas y sociales y el quepone el eje en las consecuencias para la salud de la participación comunitaria. El peso que cada criterio recibe en la argumentación y la forma en que ambos se articulan en la misma permiten organizar al interior de las categorías de aceptacion y rechazo diversas concepciones sobre la participación comunitaria identificadas en la literatura.

Dentro de la posición favorable a la inclusión de la participación comunitaria encontramos tres concepciones básicas: 1) la que privilegia en su argumentación los aspectos políticos y sociales de la participación, concibiéndola como una actividad necesaria por sus efectos democratizadores sobre la sociedad; 2) la que privilegia en su argumentación las consecuencias para la salud de problación, concibiendo a la participación como una estrategia que brinda mayores posibilidades de éxito a los programas; y 3) la que bajo una argumentación de apoyo a la participación por sus efectos en el nivel de la salud esconde una intención de manipulación en el nivel de lo social y lo político.

El primer enfoque concibe a la participación comunitaria como una forma de resolución de los problemas de salud que, al ser autogestiva, brinda a la comunidad autosuficiencia e independencia, aumentando su nivel de autonomía. Resolver de esta manera los problemas de salud tiene un "efecto de demonstración" y significa un aprendizaje para la resolución de otros problemas que aquejan a la comunidad. De Roux et al. (1990) señalan que "la participación social así definida, tiene implicaciones políticas que rebasan el marco de la atención a la salud, por cuanto significa el ejercicio de poder y como tal, (el) fortalecimiento de la sociedad civil y de la democracia de base (...), (lo que) constituye una finalidad en sí misma si se la considera como una condición inherente a la democracia real (...) (y) equivale al proceso de reapropiación por la problación, del conjunto de instrumentos que regulan la vida social y los servicios que presta". Según esta posición, el involucramiento de la comunidad en actividades de salud tiene su objetivo último no sólo en la solución del problema de salud específico, sino en el desarrollo de la comunidad como un todo, con énfasis en la autosuficiencia y asumiendo las respuesta a las necesidades tal como las articula la misma comunidad (Winch et al., 1991). En este modelo, la participación comunitaria se plantea como un fin en sí mismo y es siempre deseable, independientemente de su impacto en la salud, siempre y cuando se eleven los niveles de organización y conciencia de la comunidad.

El segundo enfoque enfatiza en su argumentación las consecuencias de la participación en la salud de la población. Desde esta perspectiva la participación es concebida como un medio técnico, una modalidad que permite el mejor funcionamiento y aceptación de los programas de salud. Su inclusión, en este caso, respondería a diversas necesidades pragmáticas.

Siguiendo este enfoque, encontramos una amplia variedad de situaciones en donde la participación comunitaria resultaría a consejable. El argumento más utilizado señala que ante el fracaso de los programas diseñados e implementados sin tomar en cuenta a la comunidad, la participación comunitaria es una opción que garantiza mayor efectividad ya que el éxito de los programas depende de que la comunidad los sienta como algo propio, responsabilizándose por el problema y participando en su solución. También se indica como alternativa cuando las acciones exclusivamente biológicas no resuelven un problema - p.e. inexistencia de prevención por immunización para una enfermedad determinada - o cuando los recursos económicos no son suficientes para implementar otro tipo de medidas (Winch et al. 1991). En este último caso la participación comunitaria es concebida como una herranienta para reducir costos, permitiendo ampliar la cobertura de los servicios gracias al financiamiento, directo o indirecto, de los mismos beneficiarios.

La postura de Winch et al. (1991) es un buen ejemplo de la concepción de la participación como un medio técnico para la implementación de los programas. Al hablar del control de enfermedades transmitidas por vectores consideran que el enfoque más útil es aquél en donde la participación debe ser negociada entre el gobierno, el sector privado y los residentes 
de la comunidad, dando más peso a los objetivos definidos por el ministerio de salud. De esta manera plantean sus diferencias con el enfoque que denominan "de desarrollo comunitario" en el que se privilegia el punto de vista de la población y que parece equivalente a la postura que plantea a la participación como un fin en sí mismo.

Otro ejemplo lo constituye la posición de la OMS expresada en la declaración de Alma Ata (1978). Según ésta, la participación comunitaria se basa en dos axiomas:

1)que el único camino para superar las limitaciones y la inequidad en la distribución de los recursos para asegurar la meta de Salud para Todos es el involucramiento de las comunidades locales en la prevención y el tratamiento, $\mathrm{y}$

2)que sin el involucramiento y responsabilidad de la comunidad, los programas fracasarán.

Así concebida, la participación comunitaria deviene en una estrategia para maximizar la accesibilidad y disponibilidad de los servicios (Manderson et al., 1991).

Esta concepción de la participación comunitaria como herramienta se piensa a sí misma como social y políticamente "neutra". La participación es sólo un medio para llegar a un fin: el mejoramiento de la calidad de salud de la población a la que se dirige el programa. Al dar todo el peso a la dimensión sanitaria, no toma en cuenta los efectos del programa sobre las dimensiones políticas y sociales relevantes para la comunidad. Pero la participación nunca es absolutamente neutra, ni puede ser abstraída del contexto en el que tiene lugar; siempre tiene algún efecto sobre la estructura social de la comunidad, su organización y su capacidad de acción. Aún cuando el objetivo último del programa se dirija exclusivamente a incidir sobre problemas de salud específicos y los beneficios que se obtengan sean claros y evidentes, al concebir a la participación como una estrategia sin consecuencias sociales, se corre el peligro de que el programa tenga efectos negativos sobre otros aspectos de la vida comunitaria. Puede conducir a consecuencias no esperadas ni deseadas por los diseñadore del programa; puede estimular la apatía y la desmovilización o incrementar la dependencia de la asistencia externa, resultando, en última instancia, contraproducente para la calidad de vida de la población (Gwatkin et al., 1979).

El discurso que ignora las consecuencias políticas y sociales de la participación comunitaria tiene además el peligro de esconder a la tercera de las posiciones señaladas, en donde la participación esté siendo incorporada en el programa como excusa para manipular políticamente a la población.

Cuando los programas tienen objetivos que van más allá de la resolución de los problemas de salud de la población como, por ejemplo, legitimar a quien lo promueve e implemente (Testa, 1990) - desde el Estado hasta grupos religiosos -, la participación comunitaria es concebida e implementada como un instrumento político. Para quienes comparten esta perspectiva, inclusive las mejoras en la salud constituirían un medio antes que un fin en sí mismo, y la participación comunitaria se incluiría como estrategia política, antes ue como herramienta de utilidad técnica. A diferencia del primer enfoque - donde también las consecuencias políticas son privilegiadas frente a las de la salud - en este caso los efectos que se persiguen pueden, incluso, ser ajenos a los intereses de la población. Obviamente, quienes incluyen a la participación comunitaria con estos objetivos, rara vez lo explicitan en su discurso y tienden a justificar a la participación por su utilidad técnica para los programas.

El segundo gran grupo de enfoques es el de aquellos que rechazan a la participación comunitaria. Aquí también encontramos argumentos distintos, de acuerdo al criterio que se privilegia para definir esta posición.

En primer lugar, en esta línea se encuentran quienes interpretan a la participación comunitaria como una excusa para manipular política y socialmente a la comunidad. Su concepción coincide con ele último enfoque que acabamos de describir - la participación como medio político - pero difiere diametralmente en las conclusiones a las que arriba: en lugar de justificarla por su utilidad, cuestionan la inclusión de esta estrategia en los programas por los efectos negativos que tiene sobre la comunidad. Ugalde, por ejemplo, analiza el sentido que los programas de participación comunitaria han 
tenido en América Latina, y concluye que la misma produjo una explotación adicional de los pobres por extracción de trabajo no remunerado. Además, contribuyó, por medio de la manipulación, al empobrecimiento de su cultura, erigiéndose en mecanismo de control y cooptación de líderes (Ugalde, 1985). Así definida, la participación comunitaria sería sólo un instrumento para legitimar al poder, ejercer control político y beneficiar intereses ajenos a los de la población. En su versión más radical, esta concepción critica indiscriminadamente cualquier inclusión de la participación comunitaria en los programas de salud, dándole a la diversidad de planteamientos con que se ha llevado a la práctica, un mismo significado.

En segundo lugar encontramos la concepción que rechaza la inclusión de la participación comunitaria por considerar que las consecuencias para la salud de los programas que utilizan esta estrategia son negativas, ofreciendo servicios de segunda clase y/o de baja calidad, con personal probemente entrenado $\mathrm{y}$, en general, con menores recursos materiales y humanos que los servicios que se ofrecen a los sectores privilegiados.

Si bien en cada una de estas dos posturas, la argumentación para rechazar a la participación se sostiene en uno sólo de los dos criterios, por lo general ambos planteamientos se presentam entrelazados: la postura que sostiene Ugalde, por ejemplo, rechaza a la participación por considerarla negativa tanto en el nivel político y social como en el nivel de las consecuencias para la salud.

Es interesante notar que, en realidad, esta posición es esclava de una lógica inductiva: a partir de la crítica a programas determinados se concluye que inevitablemente todos los programas con participación comunitaria tienen efectos negativos sobre la comunidad. La crítica a programas concretos deviene, así, en una posición maniquea, en donde se rechaza a la participación en sí misma y no a formas concretas de proponerla, planearla e implementarla.

Una tercera posición es la que rechaza a la participación argumentando que como los programas de salud con enfoque participativo tienen que ser "locales" y diferentes de una región a otra, aumentan sus costos y disminuye su factibilidad. Esta postura sería el reverso de la que apoya a la participación por su efectividad para lograr los objetivos de los programas, considerando que las dificultades son mayores que los beneficios y que, en última instancia, obstaculizan la extensión de la salud a la población.

La distancia que asumen las posiciones resultantes de cada uno de los dos criterios básicos que utilizamos para clasificar a las distintas posturas permiten que un mismo fenómeno sea interpretado de manera distinta. En el nivel de las consecuencias políticas de la participación, por ejemplo, la construcción de una clínica llevada a cabo por organizaciones democráticas de los miembros de una comunidad puede ser interpretada como: a) una conquista para romper con viejas prácticas como el paternalismo (Castellanos, 1979); o, b) como una explotación del trabajo de la comunidad para eludir obligaciones que debe cumplir el Estado (Ugalde, 1985).

Lo paradójico de esta situación es que ambas interpretaciones se originan en la misma corriente política: desde la izquierda se argumenta a favor y en contra de la participación en función de los mismos objetivos de mejorar las condiciones de vida de los grupos menos favorecidos. Evidentemente, la construcción de la clínica puede ser tanto una conquista como una explotación de la comunidad, dependiendo de la forma en que la participación se implementa en la práctica. No es cierto que cualquier forma de participación trae implícitas ventajas para la organización política y social de la comunidad, ni tampoco que genera necessariamente consecuencias negativas para la misma. De hecho, ambas concepciones aceptam, en algún grado, que las consecuencias políticas y sociales de la participación comunitaria pueden ser - aunque sea excepcionalmente - distintas a como las imaginan y que no existe necesariamente una relación biunivoca entre la participación y sus efectos.

La postura favorable a la participación critica a programas específicos donde ésta tiene un significado distinto al propuesto. Esta posición implica distinguir dos niveles: qué es y qué debe ser la participación comunitaria. Por su parte, la postura contraria a la participación también acepta que ésta puede ser positiva en determinadas situaciones. Por ejemplo, aún 
cuando sostenga que la participación comunitaria en sociedades estratificadas es sinónimo de explotación, Ugalde rescata las experiencias de participación de Cuba y Nicaragua.

Algo similar sucede cuando comparamos las posiciones extremas en la dimensión de las consecuencias para la salud: hay ejemplos que sustentan la posición que ve a la participación como una forma de extender la salud a toda la población, y también los hay para sustentar la que la concibe como una forma de brindar servicios de bajo nivel a los grupos desfavorecidos socialmente. Inclusive, podemos encontrar situaciones que expresen ambos extremos simultáneamente. Este es el caso cuando los servicios que ofrece el programa son de baja calidad en relación a otros servicios existentes o a las posibilidades que ofrece la tecnología disponible, pero aún así representa una ventaja para la población frente a la situación sobre la que se interviene.

La relación entre la participación comunitaria, sus consecuencias sociales y políticas y sus efecto sobre la salud dependen de las formas que la participación adquiera en la práctica. Es necesario, por tanto, tener claro, cuando se propone incluir a la participación en programas de salud, cuáles son los efectos que se espera tenga la participación y cómo se va a implementar para que, efectivamente, tenga los efectos esperados y exista correspondencia entre la concepción y la práctica de la participación.

Desde nuestra perspectiva, es importante considerar que la participación comunitaria tienen tanto efectos sobre los programas en términos de sus resultados en el área de la salud de la comunidad como sobre la vida social y política de la misma, y ambos aspectos deben ser considerados al evaluar su inclusión en los programas. En ninguno de los dos niveles la participación es neutra, y en ambos puede tener efectos positivos o negativos. Por ello creemos que la participación comunitaria debe incluirse en los programas cuando cumpla simultáneamente con dos objetivos explícitos: debe ser efectiva desde el punto de vista de la salud y positiva desde el punto de vista de la vida social de la comunidad. Esto implica, para la primera dimensión, que la participación comunitaria debe formar parte de los programas cuando la población reciba como resultado claros beneficios de salud y, para la segunda, que debe resultar una experiencia enriquecedora en términos del aprendizaje para resolver de forma autogestiva otras necesidades de la comunidad.

Un último aspecto a considerar en términos de la conceptualización de la participación comunitaria nos parece de crucial importancia: en la decisión de incluir o no dentro de un programa de salud a la participación comunitaria no participa la propia comunidad. Hay pocas excepciones a esta regla y una de ellas es la experiencia relatada por McKnight (1978) en Chicago, donde es la comunidad quien solicita el programa. En este caso, una orgainzación comunitaria de un barrio de población negra de bajos recursos, decidió tomar el control sobre los dos hospitales de la zona que les negaban el servicio. Después de varios años de ejercer este control, decidieron evaluar los resultados de su acción encontrando que no había habido cambios signficativos como consecuencia de la misma. A partir de estos resultados, la organización solicitó a un centro de investigación un estudio que permitiera explicar lo que había sucedido y que los orientara sobre cuáles podían ser medidas efectivas para mejorar la salud de los miembros de la comunidad. El estudio señaló como principales causas de hospitalización a una serie de problemas - accidentes automovilísticos, ataques interpersonales, otros accidentes, malestares respiratorios, alcoholismo, drogadicción y mordeduras de perros que, en realidad, eran en su mayoría problemas sociales antes que enfermedades y que, por ello, excedían al buen funcionamiento de los hospitales. En consecuencia, la organización comunitaria llevó a cabo un cierto número de acciones para combatir las causas de la hospitalización. Entre éstas, destacan la captura de perros callejeros, cambios en la vialidad para evitar accidentes y la construcción de invernaderos en los techos para el cultivo de hortalizas que mejoraran su nutrición. Varios fueron los resultados de estas actividades. En primer lugar, la gente comprendió que sus acciones cotidianas, antes que el funcionamiento hospitalario, determinan su salud. Por otro lado fortalecieron a su organización, involucrando a más miembros en sus actividades y, por último, la construcción de los invernaderos se reveló útil no sólo para 
solucionar el problema de salud, sino también otros problemas económicos y sociales, al involucrarse en su cuidado a los ancianos del asilo de la comunidad.

Sin embargo, con mucha mayor frecuencia, especialmente en Latinoaméric, la decisión sobre la participación comunitaria se decide "desde fuera", y la participación que se solicita está frecuentemente sujeta a un programa predefinido (Manderson et al., 1991).

De Roux et al. (1990) señalan al respecto que si la participación social en su sentido más amplio es un proceso complejo inherente a la dinámica de toda sociedad, la idea de que la participación es un proceso susceptible de estimularse desde afuera, y que la mayor o menor movilización de la comunidad puede ser manipulada por agentes externos, es un concepto relativamente moderno.

Para compensar, de alguna manera, la situación paradójica que se presenta cuando la participación comunitaria no es propuesta por la comunidad, varios autores sostienen la necesidad de transferir conocimientos de salud hacia la población. De esta manera su capacidad de decisión sobre la participación en los programas tendría un real sustento técnico (Manderson et al., 1991). Esto no significa convertir a toda la población en médicos, ni que cada decisión respecto a la salud deba ser tomada en una asamblea (Castellanos, 1979). Los conocimientos a transferir deben ser operativos, permitiendo a la comunidad definir sus prioridades al tiempo que se reconoce la validez de su punto de vista y de su percepción e interpretación de los fenómenos de salud como ingrediente básico para la elaboración de una visión coletiva y se articula el saber popular con elementos del pensamiento científico y técnico (De Roux et al., 1990).

\section{FORMAS DE LA \\ PARTICIPACIÓN COMUNITARIA}

La segunda dificultad para definir a la participación comunitaria es que, además de la variedad de conceptualizaciones coexistentes, las formas que adopta en la práctica son múltiples y diversas. Estas pueden ser clasificadas según características correspondientes a distintas dimensiones, entre las cuales destacan las siguientes:

a)el aspecto, etapa o momento del programa en el cual la comunidad participa;

b)los mecanismos a través de los cuales se propone la participación;

c)el horizonte temporal de la participación; y d)el tipo de acciones que realiza la comunidad.

En la primera dimensión encontramos distintas combinaciones de una variedad de estrategias posibles. Así, la participación puede darse (Manderson et al., 1991).

- sólo en la discusión;

- en la toma de decisiones en varios niveles (desde la definición de los mecanismos para el involucramiento de la comunidad, hasta el manejo de recursos);

- en el entrenamiento;

- en la ejecución de las tareas;

- sólo en la utilización de los servicios.

En términos de los mecanismos una primera distinción puede encontrarse entre los programas que proponen a la participación de individuos aislados y los que buscan que los miembros de las comunidades realicen acciones grupales a través de organizaciones. El primer modelo da origen a la clásica figura del "promotor de salud" con dos opciones: que los "promotores" sean elegidos por la comunidad o por los responsables del proyecto. El segundo modelo propone el no menos popular "comité de salud" que puede crear nuevas instancias por y para el programa de salud, o puede apoyarse en instituciones existentes en la comunidad.

En relación al horizonte temporal, encontramos diferencias entre los programas que proponen una participación permanente y aquellos donde la participación se circunscribe a períodos de tiempo limitados.

Por último, en términos del tipo de acciones que realiza la comunidad, la variedad es enorme y depende de los objetivos del programa: hacer letrinas, vacunar a los niños, distribuir medicamentos, etc.

Tres criterios aparecen como los más importantes para optar por alguma de las alternativas 
- o sus posibles combinaciones - señaladas para cada dimensión:

a) el tipo de concepción sobre participación comunitaria;

b) los objetivos específicos del programa, y

c) las características sociales, culturales, políticas y económicas de la comunidad donde éste se lleve a cabo.

Si bien los tres criterios actúan simultáneamente, tienen distinto peso para definir la toma de decisiones sobre cada dimensión. Algunos ejemplos de programas concretos nos permitirán ilustrar su relación con la clasificación propuesta.

En primer lugar, la concepción de participación comunitaria que compartan los diseñadores del programa parece tener un fuerte lugar en la decisión sobre el aspecto en que se solicita la participación de la comunidad: la postura que plantea a la participación como una necesidad en sí misma por sus efectos democratizadores sobre la sociedad, tiende a proponer el involucramiento de la población en el mayor número de aspectos, mientras que quienes la conciben de una manera más pragmática, tienden a proponerla de forma más limitada.

Un ejemplo del primer caso lo constituye el programa para la erradicación de diarrea infantil llevado a cabo en La Pampa, Argentina. En este programa, con el objeto de "aproximarse al conocimiento y experiencias con que los sectores populares enfrentan y resuelven los problemas cotidianos de la salud y la enfermedad, la percepción de la diarrea y las formas de autoayuda y de solidaridad" y de buscar "el fortalecimiento de las acciones existentes para el desarrollo del cuidado de la propia vida y dela comunidad", se involucró a la población en el diagnóstico del problema, la elaboración de propuestas para la transformación de situaciones concretas y en la realización de las acciones (Di Liscia \& Novacovsky, 1988).

La segunda situación puede ejemplificarse con un programa de administración masiva de medicamentos realizado en Kenia para controlar la filariasis. En este caso, la distribución de los fármacos se realizó a través de personal local no médico, como una estrategia para conseguir mayor aceptación del programa y reducir los costos resultantes de que las tareas fueran reali- zadas por personal con entrenamiento médico (Wijers \& Kaleli, 1984).

La relación entre concepción de participación comunitaria y propuesta concreta de la misma, dista mucho, sin embargo, de ser biunivoca y su correspondencia no siempre resulta tan clara. Encontramos casos en los que la participación es indicada exclusivamente como una herramienta para asegurar los resultados de los programas y en los que es muy amplia, y otros en donde el discurso de la participación no corresponde con la forma que ésta adquiera en la práctica. Un ejemplo del primer caso es el programa de control de malaria llevado a cabo en Pondicherry, India, donde se ayudó primero a solucionar problemas considerados como apremiantes por la población - agua potable y accesso a la atención médica - para obtener la aceptación y participación de la comunidad en el programa (Rajagopalan \& Panicker, 1984). Un ejemplo del segundo caso lo constituye una experiencia realizada con los mineros de Real del Monte y Pachuca en México, donde detrás de un discurso sobreideologizado sobre el trabajo conjunto, los obreros se limitaron a recibir atención médica y aceptar algunas sugerencias de incluir en sus demandas cuestiones relacionadas con la salud (Gutiérrez, 1981).

La concepción sobre la participación comunitaria también influye sobre la elección del mecanismo para lograrla. Por ejemplo, Gonzalez de la Torre (1984), a partir de su experiencia en la implementación de programas de salud en Chiapas, México, sostiene que el comité de salud es una instancia preferible al promotor de salud, ya que evita la acumulación de conocimientos en una sola persona - con los consecuentes abusos potenciales que ésto puede significar - y permite la participación activa y crítica de la problación en los programas.

La postura que privilegia las consecuencias políticas y sociales de la participación, tiende a apoyar, como consecuencia lógica de su concepción, la promoción de la participación a través de organizaciones ya existentes en la comunidad. En el caso en que se creen comités de salud o se opte por promotores de salud, esta postura se inclina porque su elección sea realizada democráticamente por la población.

Sin embargo, tampoco en este caso la relación entre concepción de la participación y mecanismo propuesto es mecánica y es posible llegar a 
conclusiones similares a las expuestas desde una perspectiva más pragmática, que busque el mejor modelo para asegurar el éxito del programa.

En una evaluación sobre distintas experiencias de participación comunitaria en América realizado por la OPS, el trabajo de Colombia concluye, por ejemplo, que es preferible el uso de organizaciones comunitarias existentes como mecanismos para la participación comunitaria, ya que imponer la formación de un sistema de comités de salud comunitarios y municipales jerárquicos puede restringir la participación a pequeños grupos y crear problemas para integrar a la comunidad en proporciones más amplias. Este mismo trabajo señala que el hecho de que la mayoría de los promotores de salud sean pagados y supervisados por el sistema de salud, aún habiendo sido seleccionados de y por la comunidad, tienden a separarlos de la comunidad y a identificarlos totalmente con el sistema formal: se convierten en el personal de salud de más bajo nivel antes que en un vínculo con la comunidad. El texto concluye que para que el promotor de salud sea un lazo efectivo con la comunidad, éste debe ser responsable, en primer lugar, ante la comunidad. Aún cuando el sistema de salud provea la supervisión técnica, la comunidad debe ser capaz de evaluar el desempeño del promotor y reemplazar a un promotor inefectivo (PAHO, 1984).

El segundo de los criterios mencionados las características del programa de salud en cuestión - también ocupa un lugar importante en la difinición del mecanismo de participación más apropiado. En varios países de América Latina, por ejemplo, se han puesto en prática programas de capacitación para parteras empíricas (PAHO, 1984). Evidentemente, en este caso la participación se restringe a individuos que cumplen con funciones determinadas en la comunidad. La creación de un comité no tendría mucho sentido.

Por último, las características de la comunidad donde se desrrolle el programa hacen a determinados mecanismos más factibles que a otros. Por ejemplo, la comunidad puede no contar con organizaciones activas, por lo que crear organizaciones por iniciativa del programa puede resultar la única alternativa.

En términos de definir la temporalidad de la participación y el tipo de tareas que se solicita a la comunidad, uno de los criterios con mayor peso es el tipo de programa de salud del que se trate.

Con respecto a la primer característica, podemos identificar programas de largo plazo, que requieren instancias de participación más permanentes - como el de Salud Comunitaria implementado en Panamá, donde la participación se realiza a través de "Comités de Salud" establecidos en cada comunidad (La Forgia, 1985) - y aquéllos que tienen objetivos más concretos, y son proyectos a más corto plazo, como los de control de enfermedades tropicales, donde la participación se circunscribe a un período de tiempo limitado.

Las tarea y acciones emprendidas por la comunidad también varían acuerdo al tipo de problema de salud que se pretende solucionar. Por ejemplo, Gordon (1988) argumenta que en el caso del dengue, los recipientes de agua artificiales proveen un lugar ideal para la reproducción del vector, por lo que la participación debe ser orientada a los esfuerzos individuales de las amas de casa para eliminar estos sitios requiriéndose estrategias educativas para modificar conductas individuales antes que alentar actividades comunales. En contraste, este autor señala que los proyectos para control de la malaria requieren programas de participación colectiva, porque los sitios de procreación del vector son espacios comunes y públicos, como lagunas y charcos, en donde las actividades deben ser llevadas a cabo por instancias organizadas de miembros de la comunidad.

Por último, las características sociales, culturales, económicas y políticas de la comunidad también definen las posibilidades y formas de la participación. Por ejemplo, en Cuba, la situación postrevolucionaria implicó una mayor participación de la comunidad en el sistema de salud en general (PAHO, 1984). La participación también puede ser moldeada por los eventos cotidianos de la comunidad, por ejemplo, puede tener menor envergadura en los períodos de mayor actividad agrícola. Pero sobre todo, 
las características de la comunidad afectan los resultados que tenga en la práctica el modelo de participación comunitaria propuesto por un programa de salud determinado.

Así como la relación entre la concepción sobre la participación comunitaria y la propuesta concreta de la misma no es biunivoca, tampoco hay una correlación clara entre distintos modelos y sus resultados. La historia de éxitos en conseguir metas a través de la acción comunitaria, la existencia de estructuras sociales a través de las cuales la participación puede ocurrir, el nivel de educación, la concentración espacial y el transporte, la presencia de líderes, son algunas de las características comunitarias que parecen favorecer la práctica de la participación. Por el contrario, la falta de homogeneidad social, profundas divisiones económicas y políticas y la creencia de que la salud es sólo terreno de expertos o que la responsabilidad recae exclusivamente en el Estado, son algunas de las características que parecen obstaculizar las posibilidades de la participación comunitaria.

\section{EL PAPEL DE LA INVESTIGACIÓN}

Una premisa ampliamente aceptada es la necesidad de adaptar los programas a las características sociales, culturales, económicas y políticas de cada comunidad como condición para su desarrollo exitoso. Esto implica tener un amplio conocimiento sobre la comunidad para poder tomar las decisiones adecuadas y pertinentes. De otra manera, inclusive las consideraciones metodológicas pueden parecer contradictorias: ¿es conveniente respetar la organización de la comunidad, fortaleciendo y consolidando sus propias instancias para la participación (Castellanos, 1979), o — como se sugiere en otros casos - no reforzar estructuras de explotación existentes brindando ayuda a través de quienes controlan las organizaciones, legitimando y beneficiando sólo a los más poderosos (Gwatkin et al., 1979; PAHO, 1984)?

Adaptar y modificar los programas a la realidad comunitaria, tomando en cuenta, ademas, el contexto regional, nacional e internacional donde ésta se sitúa requiere partir de una investigación previa al diseño del programa.
En la metodología de las ciencias sociales ha habido una larga discusión sobre las ventajas y desventajas de las estrategia cualitativas y cuantitativas y sobre las ventajas de unas $u$ otras en relación a los objetivos de la investigación.

Sin embargo, hay un aparente consenso sobre la utilidad de las técnicas cualitativas en las investigaciones orientadas a lograr la adaptación de los programas de salud a las características particulares de cada comunidad (Winch \& Hudelson, 1991; PAHO, 1984) ya que éstas permiten aprehender los procesos y las complejas relaciones que constituyen el cuerpo social. Los trabajos de Scrimshaw \& Hurtado (1987) y Chamber (1985) son buenos ejemplos de cómo puede utilizarse estas herramientas.

Las técnicas cualitativas permiten obtener la información necesaria para el diseño del contenido y de la forma de los programas. En cuanto al contenido, al reconstruir la percepción y representación social de la enfermedad, los conocimientos, las prácticas y experiencias y las rescatar el lenguaje propio de la comunidad, permiten estabelecer um diálogo auténtico sobre el problema. En cuanto a la forma, al brindar datos sobre los tipos de organización, las costumbres y la dinámica de la comunidad, permiten proponer un tipo de participación comunitaria coherente con el contexto. Simultáneamente, la información sobre la vida social de la comunidad permite plantear una forma de participación comunitaria que, más allá de los objetivos del programa, resulte una experiencia auténticamente enriquecedora para todos los miembros de la comunidad.

Las ciencias sociales al incorporarse a los programas de salud con participación comunitaria no deben limitarse sólo a aportar los datos necesarios para la mejor instrumentación de la misma como estrategia de acción (De Almeida \& Silva, sin fecha); deben brindar también una interpretación sobre la forma en que el programa afecta la productividad y la estructura social de la comunidad. Además de las contribuciones que puedan realizar par una efectiva adecuación de los programas a las condiciones locales, deben vincular los problemas de salud con el contexto global de la comunidad, y evaluar el impacto que sobre ésta puede tener un tipo de participación determinada. 


\section{COMENTARIOS FINALES}

La revisión de la literatura sobre participación comunitaria nos permite concluir que bajo el mismo nombre se agrupan fenómenos muy heterogéneos. Para comprender su diversidad necesitamos, en primer lugar, distinguir la existencia de distintos niveles: la concepción global sobre la utilidad y pertinencia de la participación comunitaria, el modelo de participación propuesto y la forma en que este modelo es lleva a cabo en la práctica. En cada uno de estos niveles encontramos, como hemos descrito, diferentes posiciones y propuestas. Esto dificulta la posibilidad de realizar una evaluación global sobre el uso de este recurso en los programas de salud, obligando, antes de realizar cualquier tipo de consideración, a caracterizar de qué tipo de participación se trata.

Aún así, conviene hacer algunos comentarios sobre ciertos problemas generales que se presentan independientemente de la posición adoptada para cada uno de los niveles señalados. Estos se refieren al paso de un nivel a otro y a la articulación entre niveles. Como se analizó en el trabajo, concepciones distintas sobre la participación pueden proponer modelos de participación semejantes y, por el contrario, concepciones semejantes pueden resultar en modelos muy distintos. Aún cuando, como hemos visto, existe una importante relación entre ambos aspectos, la naturaleza de la relación es frecuentemente poco clara o directamente confusa. Sólo si las propuestas hacen explícita su concepción sobre participación comunitaria y el proceso por el cuál optaron por un determinado modelo de participación puede evaluarse la coherencia con lo que realizan en la práctica.

Por otro lado, cuando se trata de adaptar modelos generales a programas y situaciones concretas, las dificuldades emergen con más fuerza. Aún cuando existe cada día mayor reconocimiento del papel que la investigación sobre las características sociales, culturales, políticas y económicas de la comunidad puede ofrecer para la implementación de los programas, es todavia necesario el desarrollo de estrategias que faciliten la utilización de los datos generados por estos estudios, especial- mente cuando van a ser utilizados por personal formado en disciplinas distintas a las ciencias sociales.

A pesar de la variedad de experiencias existentes, continúan sin respuesta algunas preguntas básicas sobre la forma operativa de implementar la participación comunitaria en los sistemas nacionales de salud: ¿Cómo solucionar las contradiciones existentes entre la orientación y estructura de la mayoría de los sistemas de salud gubernamentales, y las condiciones necesarias para la participación comunitaria?; ¿cómo conciliar las prioridades comunitarias con las de los profesionales de la salud cuando éstas no coinciden?; ¿cómo trasladar experiencias piloto exitosas a programas regionales o nacionales (Gwatkin et al., 1979; PAHO, 1984)?

Por sobre todas las otras, la pregunta esencial que hay que responder es acerca del carácter necesario dela participación comunitaria en el mejoramiento de la calidad de la salud de los habitantes. A pesar de ser señalada como elemento clave para el éxito de los programas de salud, la mejora del nivel de salud de la población como consecuencia de la participación comunitaria continúa siendo un resultado ambiguo y dudoso (Ugalde, 1985; De Roux et al., 1990). De hecho, puede no ser necesaria o indicada en todos los casos. Esta situación refleja uno de los problemas que aparece en la mayor parte de los trabajos revisados la falta de criterios claros para evaluar los resultados de los programas de salud, y si éstos pueden interpretarse como efecto de la participación comunitaria (Gwatkin et al., 1979). Altman sugiere que la pregunta básica sobre la efectividad del programa, debe dividirse en preguntas más significativas y concretas. Este autor propone que la evaluación debería hacerse sobre tres aspectos diferentes: el proceso del programa, los efectos fisiológicos, psicológicos y sociales y la relevancia social del mismo en términos de costo-efectividad y utilización y generalización de los resultados (Altman, 1986). Podemos agregar, a estos aspectos, la necesidad de evaluar el éxito de la participación comunitaria en sí misma, más allá de su impacto sobre la salud, examinando sus efectos en la vida social de la comunidad, analizando sus consecuencias para promover la autonomia y la 
democratización. El desarrollo de técnicas de evaluación adecuadas es uno de los retos importantes a los que deben responder los programas.

El segundo reto, si finalmente se concluye que la participación comunitaria debe formar parte de los programas, es buscar cómo implementar una participación que realmente responda a la propuesta con la cual se la incluye en los programas, es decir su coherencia. Las experiencias de participación exitosas señalan que cuando la participación comunitaria se propone respetando la estructura organizativa de la comunidad y brindando posibilidades de crecimiento a sus miembros, pueden alcanzarse altos grados de participación e involucramiento. La salud no es un fenómeno aislado de la realidad comunitaria. Cuando la propuesta de participación integra los objetivos en salud con beneficios en otras áreas de la vida de la comunidad, ambos aspectos se enriquecen mutuamente, ampliando las posibilidades de éxito de los programas.

Sin embargo, a pesar de que éstas son indicaciones generales que orientan la dirección que deben tomar futuros trabajos, quedan aún sin responder las preguntas de carácter operativo. Es necesario trabajar con mayor intensidad en los aspectos relacionados con la implementacion de los programas, recuperar experiencias y buscar estandarizar, hasta donde sea posible, una metodología que garantice la mayor correspondencia entre la intenciones y los resultados.

\section{RESUMEM}

BRONFMAN, M. \& GLEIZER, M. Participación Comunitaria: Necesidad, Excusa o Estrategia? O de qué Hablamos Cuando Hablamos de Participación Comunitaria. Cad. Saúde Públ., Rio de Janeiro, 10 (1): 111-122, jan/mar, 1994.

El presente trabajo evalúa la estrategia de incluir a la participación comunitaria en los programas de atención y promoción a la salud, considerando tres dimensiones: la pertinencia de la prática, las características de su impementación y los resultados que genera.
Realizando una revisión crítica de la bibliografia sobre el tema, se llega a la conclusión de que bajo el término "participación comunitaria" se agrupan propuestas muy heterogéneas. Esto obliga, antes de cualquier consideración, a especificar de qué tipo de participación comunitaria se trata. Se propone una clasificación de las diferentes prácticas que se agrupan bajo este nombre, según los siguientes criterios: 1) Las concepciones teóricas e ideológicas que la sustentan, 2) los aspectos en que se propone implementar y las áreas sobre las que pretende influir y 3) las modalidades que adopta en la práctica. Se analizan la articulación existente entre estos tres aspectos. Por último, se considera el papel de la investigación social y de la metodología cualitativa en la implementación de la participación comunitaria en programa de atención a la salud y se examinan los retos que debe enfrentar la propuesta general más allá de sus diferencias de concepción e implementación - para que la estrategia se desarrolle exitosamente.

Palavras-Chave: Participación Comunitaria; Programas de Promoción a la Salud

\section{REFERÊNCIAS BIBLIOGRÁFICAS}

ALTMAN, D., 1986. A famework for evaluating community-based health disease prevention programs. Social Science and Medicine, 22: 479487.

CASTELLANOS, P., 1979. Reflexiones sobre la Medicina Comunitaria y la Participación de la Comunidad. Caracas: Escuela de Medicina "José M. Vargas", Universidad Central de Venezuela. (Mimeo.)

CHAMBERS, R., 1985. Shortcut methods of gathering social information for rural developmen projects. In: Putting People First (M. Cernea, ed.), pp. 399-415, New York: Oxford University Press.

CONIL, E., s/d. A Noção de Saúde Comunitária: Elementos de comparação internacional. Campinas. (Mimeo.)

DI LISCIA, M \& NOVACOVSKY, I., 1988. Participación comunitaria para la erradicación de la diarrea infantil. Cuadernos Médicos Sociales, 1988: 61-68. 
DE ROUX, G.; PEDERSEN, D.; PONS, P. \& PRACILIO, H., 1990. Participación social y sistemas locales de salud. In: Los Sistemas Locales de Salud (OPS, org.), pp. 28-49, Washington: OPS.

DE ALMEIDA, N. \& SILVA, J., s/d. Sociocultural Problems of Health Programs or Resistance to the Extension of Health? North Carolina: Department of Epidemiology, School of Public Health, University of North Carolina. (Mimeo.)

GONZALEZ DE LA TORRE, E. 1984. ¿Promotor de salud o comité de salud? II Conferencia Interamericana de Educación Para la Salud, México: Unión Internacional de Educación para la Salud, Secretaría de Salubridad y Asistencia (México) y OPS.

GORDON, A. J., 1988. Mixed strategies in health education and community participation: An evaluation of dengue control in the Dominican Republic. Health and Education Research, 3: 399-419.

GUTIERREZ, I., 1981. Trabajo de Salud de los Mineros, con los Mineros. Conference International Group for the Study of the Political Economy of Healt, San Miguel, Regla, México: Universidad Autónoma de Hidalgo. (Mimeo.)

GWATKIN, D.; WILCOX, J. \& WRAY, J., 1979. Can Interventions Make a Difference? The Policy Implications of Field Experiment Experience. Report to the Word Bank, Washington D.C.: World Bank. (Mimeo.)

LA FORGIA, G., 1985. Fifteen years of community organization for health in panama: An assesment of current progress and problems. Social Science and Medicine, 21: 55-65.

MANDERSON, L.; VALENCIA, L. \& THOMAS, B., 1991. Bringing the people. In: Community Participation and the Control of Tropical Diseases. Resource Papers for Social and Economic Research in Tropical Diseases $\mathrm{N}^{\circ} 1$, UNDP/ WORLD BANK/WHO/TDR, Geneve: World Health Organization.

McKNIGHT, J., 1978. Community Health in a Chicago Slum. (Mimeo.)
PAHO (Pan American Health Organization), 1984. Community Participation in Health and Development in the Americas. An Analysis of Selected Case Studies. Washington D.C.: PAHO. (Scientific Publication $\mathrm{N}^{\circ}$ 473).

RAJAGOPALAN, P. \& PANICKER, K., 1984. Feasibility of community participation for vector control in villages. Indian Fournal of Medical Research, 80: 117-124.

SCRIMSHAW, S. \& HURTADO, E., 1987. Rapid Assesment Procedures for Nutrition and Primary Health Care: Antropological Approaches to Improving Programme Effectiveness. Los Angeles: UCLA Latin American Center/Tokyo: United Nations University.

TESTA, M., 1990. Pensar en Salud. Washington D.C.: OPS/OMS.

UGALDE, A., 1985. Ideological dimensions of community participation in Latin American health programs. Social Science and Medicine, 2: 41-53.

WHITE, A., s/d. Medicine for a Second Class: a Review of Ideas on Community Participation and Village Health Workers. London. (Mimeo.)

WIJERS, D. \& KALELI, N., 1984. Bancroftian filariasis in Kenya. V. Mass treatment given by members of the local community. Annals of Tropical Medicine and Parasitology, 78: 383.

WINCH, P; KENDALL, C. \& GUBLER, D., 1991. Community Participation in the Control of vector-Borne Diseases: A Review of Selected Activities in Latin America. Trabalho apresentado no Simpósio "Community Participation and Control Efforts in Developing Countries", Annual Meeting of the American Mosquito Control Association, Louisiana, 16-21 de marzo.

WINCH, P. \& HUDELSON, P., 1991. Formative Research in Community-Based Vector Control Programs. Trabalho apresentado no Simpósio "Community Participation and Control Efforts in Developing Countries", Annual Meeting of the American Mosquito Control Association, Louisiana, 16-21 de marzo. 